\title{
HUNDERT JAHRE EINES BEKANNTEN SLOWENISCHEN SPRACHWISSENSCHAFTLERS, PROFESSOR DR. KAREL OŠTIR
}

Am 27. Dezember 1973 starb in Ljubljana Professor der vergleichenden Sprachwissenschaft an der Universität Ljubljana, Dr. Karel OŠTIR. In den letzten Jahren seines Lebens hat er sich meist in privater Gesellschaft gehalten, obwohl seine Gesundheit es zuliess, ziemlich oft in den linguistischen Abteilungen der Philosophischen Fakultät zu erscheinen; er liebte nämlich lange Diskussionen über alle möglichen Dinge, Politik, Philosophie u.a., ja sogar über neueste Ergebnisse der Sprachwissenschaft selbst. Als Thema seiner Diskussionen stand da seine beliebte alarodische Theorie, allen anderen voran aber das Etruskische und die indogermanische Laryngaltheorie, augenscheinlich Erbschaft aus den ersten Dezenninen seines Lebens. Doch die Gesundheit ging allmählich unter und am erwähnten Dezembertage ist unser Professor sanft entschlafen. Eine Trauerbotschaft für uns Linguisten, die wir wussten, dass uns langjähriger Lehrer und Freund für immer verliess!

Nach Verlauf von fünfzehn Jahren wollen die Linguisten von Ljubljana eine gebührende Hundertjahrfeier veranstalten, denn er wurde am 13. 10. 1988 geboren. Von unserem sprachwissenschaftlichen Zirkel wurde beschlossen, dass sein Geburtstag mit einer feierlichen Sitzung und mit einer Gedächtnisrede gefeiert werden sollte. Diese letzte Aufgabe übernahm sein Schüler und Nachfolger Dr. Bojan ČoP.

Ich reproduziere den Text der Rede in deutscher Sprache, da die slowenische Fassung wohl zu wenigen Lesern der Linguistica verständlich wäre. Hier unten sollte ich also eine möglichst freie Übersetzung des Redewortlautes bieten, weil in den letzten 20 bis 30 Jahren manche Richtung der historischen Sprachwissenschaft recht merkbar entwickelt und erweitert wurde, besonders auf den Gebieten, für die OŠTIR ganz speziell interessiert war. Eine solche Diskrepanz könnte Schaden bringen; genügend analysierte Fragen findet der Leser in Linguistica XIII (Ljubljana 1973), SS. 1-96. Zur alarodischen Sprachwissenschaft heute vgl. B. ČOP, Linguistica XVI (Ljubljana 1976), SS. 3-33. Natürlich sind einige neue Theorien usw., wenn relevant, mit eingetragen. Alles möglichst kurz.

\section{Liebe Kollegen,}

vor genau 100 Jahren wurde Dr. Karel OŠTIR in Arnače (Št. Ilj bei Velenje, am 13. 10. 1888) in einer Bauernfamilie geboren; sein Leben verlief bis Abitur wie das der gewöhnlichen Bauernsöhne. Das klassische Gymnasium besuchte er in Celje und Maribor (Abitur 1909). Im Gymnasium zeigte er ein ausserordentliches Interesse 
und Begabung für das Studium der Sprachen, sowohl der modernen, wie der klassischen; Griechisch beherrschte er so gut, dass er in der fünften Klasse schon Thukydides las. Bald bekam er auch sprachwissenschaftliche Bücher in die Hände, darunter auch das griechische etymologische Wörterbuch von Boisacq, das er in einer Buchhandlung auslieh. Auf solche Weise studierte er Etymologien und begeisterte sich für die vergleichende Sprachwissenschaft. Leider musste er nach kurzer Zeit BoISACQ zurückerstatten, da er kein Geld hatte um es zu kaufen. Das böse Schicksal traf ihn bald von neuem: Nach Vatersplänen müsste er nach Gymnasium ins Alumnat treten, wie es so vielen slowenischen Intelektuellen -in jenen Zeiten erging. Schon war er in einem Kloster, als er - einer Idee folgend - aus einem Mönchenkleid sich ein ziviles zuschnitt und entfloh. So bewahrte er seine Freiheit, die vergleichende Sprachwissenschaft aber gewann mit ihm einen genialen Forscher und Mitarbeiter. Er flüchtete nach Graz (Gradec), wo er vergleichende Sprachwissenschaft einschrieb. Sein Professor war der damals schon berühmte Linguist MERINGER (Wörter und Sachen); als er einmal ein slawisches Akzentproblem zu erklären suchte, stand OŠTIR auf und sagte: "Herr Professor, das wird nicht stimmen". Der gütige Lehrer war ein wenig überrascht von einem Beginner so unterrichtet zu werden, jedoch lud er ihn zur Tafel, um die Akzentfrage richtigzustellen. Seit diesem Tag wurde OSTTIR mit MERINGER gut befreundet, sein Professor sorgte ein wenig auch für die materielle Seite seines Schülers (indem OŠTIR für die Zeitschrift Wörter und Sachen Indices und Ähnliches besorgte und dafür honoriert wurde). Dadurch wurde der ewige Hunger OŠTıRS (sein Vater wollte von ihm wegen der Klostergeschichte nichts mehr hören, so dass Karel manchmal nur ein paar trockene Birnen in der Tasche hatte), für immer verbannt.

Auf so originelle Weise begann die wissenschaftliche Karriere unseres Studenten. OŠTIR selbst lobte diese Studienjahre (1909-1913) als die glücklichsten seines Lebens. Der innere Schaffensdrang trieb nun OŠTIR von Graz nach Wien, wo er ähnliche Programme aus der Sprachwissenschaft einschrieb; hier war sein Lehrer der berühmte Profesor Paul KRETSCHMER, ganz andersartig im Vergleich mit MERINGER: grob und unfreundlich; gegenüber OŠTIRS Ungeschicklichkeit spöttisch, ablehnend. Auch in der Methode gab es starke Differenz: KRETSCHMER mit (nach OŠTIRS Meinung) allzu langsamer Verarbeitung des philologischen Materials, anderseits OŠTIR mit wuchtigem, schnellem Drang nach Resultat. So war das Schicksal schon wieder von OŠTIR selbst bestimmt: Das Dissertationsthema, das KRETSCHMER anbot - philologisch bearbeitete Ortsnamen von Attika, - lehnte OŠTIR energisch ab (S. weiter unten).

Von Wien aus verliess er Oesterreich und arbeitete weiter in Petrograd (Leningrad) und schliesslich in London (British Museum) kurz vor dem ersten Weltkrieg (1913-1914). Er wurde in die österreichische Armée berufen, wusste sich dem aktiven Militärdienst zu entziehen, hatte aber das Glück als Dolmetscher in einer Abteilung zu arbeiten, wo es österreichische Kriegsgefangenen gab, sogar aus Kaukasus. So kam er endlich mit echten "Alarodiern" in Kontakt. Wenn er Zeit hatte, schrieb er auf und prägte sich éin das kaukasische Sprachmaterial. Nach dem Kriege ging er 
schon wieder nach Wien (1918-1922). Unterwegs erwarb er in Graz (Anfang 1919) den Grad eines Doktor Phil. mit der Dissertation "Urindogermanischer langvokalischer Intransitiv". Anfang 1922 wurde er zum Dozenten für vergleichende Sprachwissenschaft an der Universität Ljubljana ernannt, 1924 zum ausserordentlichen, 1932 zum ordentlichen Professor gewählt. Im Jahre 1953 wurde er wirkliches Mitglied der Slowenischen Akademie der Wissenschaften und Künste, legte aber nach einigen Jahren diese Ehre ab. Er war als Professor der vergleichenden Sprachwissenschaft bis 1959, als er emeritiert wurde, tätig.

Bis zum ersten Weltkrieg wurde OSTTIR schon gut bekannt, zunächst durch kleinere indogermanistische Etymologien und grammatikalische Beiträge, dazu Baltoslovanska metatonija, Razpr. II, Ljubljana 1925, SS. 55-120. S. die Bibliographie in Lingu. XIII, SS. 9-12. Aber wohlwollendes Schicksal brachte ihn schon in Kriegszeiten in direkten Kontakt mit den kaukasisch sprechenden. So beginnt seine wahre alarodische Aera, als Beitrag zum Hauptthema in seiner Doktordiss. der Anhang "Exkurs über gr. híppos (Beitrag zur alarodischen Sprachwissenschaft)".

Über die etymologische Methode(n) OŠTIRS hauptsächlich mit auch bei anderen Forschern befindlichen Elementen Wort - Bedeutung - Phonetik - Struktur heimisch oder entlehnt? - Chronologie usw. sollen Zeugniss ablegen:

a) echt indogermanisch: geradezu genial zerlegt OŠTIR das alb. Wort kopil "uneheliches Kind" in Wörter und Sachen V (1913), S. 220 Anm. = interrog. ${ }^{*} q^{u-} \bar{a}$ - (idg. auch pejorativ) + ${ }^{*}$ pélno-s: pjell "gebäre"; als "vorbehaltlos" richtig nach JOKL. - Später hat Autor, schon bis zum Halse im alarodischen Wasser steckend, kein Bedenken getragen, kopil als alarodisch zu ir. cumal "Sklavin", zu proklamieren!

b) folgendes Wort zeugt nebenbei auch davon, wie aus schlecht bezeugtem (oder dem Etymologen vorerst unbekanntem) Wort ein "ghost-word" entsteht: In HORN, Grdr. d. npers. Etym. 196 fand ich zufälligerweise ein auch bei HESYCH (késter: neanías) sich befindliches Wort, etwa /kästär/; mir schien es sofort mittelpersisch zu sein, daneben noch pers, $k i h$, teilte meinen Professoren GROŠELJ und OŠTIR den Vorfall mit (ich war Antialarodist und wollte OŠTIRS alar. Beurteilung - etr. husiur "Junge" (VogelN. 8) widerlegen). Als wir die Stelle in HORN fanden, erwies sich kester samt kih ganz normal als Komparativ zu idg. *kak-ú-POK. 521-2.

c) sl. ryba "Fisch" gehört sicher nicht zu d. Raupe (Bedeutung!?). OŠTIR stellt ryba zu gr. orphṓs "serranus gigas" (ein Fisch) Anthr. VIII, 1913, S. 166. Das sl. Wort hat zu allgemeine Bedeutung, als dass es eine Vergleichbasis für das griechische darstellen dürfte. Die phonetische Seite beider Wörter ist leider so, dass man sogar zehn Urformen rekonstruieren könnte: sl. <

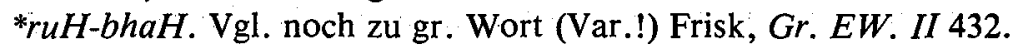

d) sicher nichtindogermanischer Herkunft ist dagegen das Wort für "Feige" in lat. ficus, gr. sỹkon, arm thuz, grus. lugi und zahlreiche Verwandte und Sy- 
nonyma. Die fast vollständige Gruppe bei OŠTIR, Btr. I (1921), SS. 1-33; er stellt sie alle in die einseitige Übersichtstabelle, s. S. 33. In dieser Tabelle hat unser Professor seine ganze Fähigkeit für phonetische und morphologische Analyse gezeigt, Eigenschaft, die samt dem Sinn für das Ganze, für das System einem wahren, reifen Wissenschaftler methodische Basis bildet.

e) noch ein anderer Typus steckt im Wort für "Blei": gr. mólybdos und lat. plumbum: man ist versucht, auch (mit $\mathrm{O}$.) ung. (ural.) ólom hier zu buchen. OŠTIR sieht in allen drei éine Wortfamilie, alar. Element. - B. ČoP hält es für mediterran. (Lingu. XIII, S. 96). Heute gilt es für ein Wanderwort, dessen Nationalität noch nicht bestimmt ist. Wichtig ist dabei, dass es auch im Uralischen vorkommt.

Die obigen 5 Beispiele sind sehr verschiedener Art: Einige sind in Wahrheit indogermanisch (alb. kopil; iran. kester sogar eine späte Glosse), noch nicht mit Sicherheit ausserhalb der Sprache gefunden (sl. ryba), sicher fremd (gr. sỹkon mit Verwandten der ganzen alten Welt; gr. mólybdos, eventuell dazu ung. ólom).

Man sieht, dass die Herkunft der alarodischen Wörter (insofern sie wirklịch alarodisch sind), tatsächlich in allen Stücken recht unsicher ist; die Alarodier waren ja im Altertum überhaupt durch nichts bekannt. OŠTIR hat die erste aller Pflichten stark vernachlässigt: den Nachweis der nationalen Angehörigkeit; die Heranziehung des Namens Urartu ist belanglos. OŠTIR wollte die Lückenhaftigkeit der geschichtlichen Tradition selbst, ohne Quelle, ergänzen. Hier zeigt sich in vielen Fällen die Schädlichkeit seines Entschlusses, wie möglich wenig Philologie zu treiben; O. war sich damals wohl dieses Mangels bewusst, die Schule von Kretschmer erschien ihm jetzt in ganz anderem Licht.

Wenn wir doch schon diese Fragen auf sich beruhen lassen, so war $\mathrm{O}$. in der Wahrheit am Misslingen seiner Thesen und Hypothesen weniger schuld als oben betont. Einiges soll doch hervorgehoben werden:

Viele "alarodischen" oder "vorindogermanischen" ("vorgriechischen" usw.) Elemente (vorwiegend Wörter, diese meist isolierte Glossen) können bei näherem Zusehen eine bessere indogermanische Etymologie erfordern: so z.B. lat. félè-s "Wildkatze; Marder", das nichtidg. sein soll (vgl. Walde-Hofmann, $L E W{ }^{3}{ }^{3}$ I 474), ist jedoch sicher eines der indoural. Wörter, also auch idg. (s B. C̆oP, $K Z$. 88 (1974), S. 50); Ähnliches auch innerhalb des Nostratischen: "Ficus" ist samt sum. ma "Feige", am besten zu finn. make- $a$ "süss" (ohne auswertige Etym.) zu stellen. Vgl. noch heth. "GIšhaššikka-.

Eine äusserliche Ursache, dass die Werke OŠTIRS mit Ungunst aufgenommen wurden, ist die äusserst gedrängte Darstellungsweise (bis 100 Etymologien pro Seite, so in zweiter Hälfte von Btr.), hat MEILLET doch in einer Kritik gesagt "prend le vertige"! 
Jeder Vergleich beruht auf Erkenntnis, dass die verglichenen Elemente, Gegenstände untereinander gewisse Ähnlichkeit/Gleichheit aufweisen, die in der sprachlichen Welt auf verschiedene Weise entstanden sind, etwa durch:

1. Urschöpfung (Nachahmung der Naturlaute u.a.); 2. Urverwandtschaft; 3. Entlehnung (aus gemeinsamer Quelle); 4. Zufall usw. - 5. phonetische Angleichung (bei Zusammenfall der urspr. verschiedenen. Laute $=$ Homonymie) usw. Alles dies untereinander gekreuzt und immer komplizierter werdend. Diese innere und äussere Differenzierung führt oft zu völliger Verschiedenheit (Fall sto "100": hundert). Die hier untersuchte Substratsprache, ALARODISCH, gilt für eine zusammenhängende Gruppe von Dialekten, die anscheinend mit dem Indogermanischen nichts zu tun hätten; dagegen spricht noch zweierlei:

a) die Existenz eines $k$-Plurals auf beiden Seiten, im Alarodischen in Namen der Stämme, z.B. Táo-kh-oi, Kardoũ-kh-oi, zu iur. (s. B. C̆OP, Dekl. pas$\operatorname{sim})$;

b) ein $n$-Suffix des "Herrschenden": lat. domi-nu-s u.a., zu sumer. geš- $t i-n$ "Wein", e-n „(Haus = e)Herr" usw.

Die Erforschung der seit Altertum östlich von Kleinasien wohnenden Völker und Stämme, vor allem der Hurriter und Urartäer, hat gezeigt, dass diese eine ostkaukasische Sprach(grupple bilden, vlg. DiAKONOFF-STAROSTIN, Hurro-Urartian as an eastern Caucasian Language (München 1986). Es ist etwas gefährlich, sich die vorindogermanischen Einflüsse so linear vorzustellen, wie so die Alarodisten meist, unter ihnen vor allem MARR, BRAUN, SCHRIJNEN und OŠTIR noch taten. S. MSL. $X X I I I(1927)$, SS. 53ff.

Mann muss nämlich vor Augen halten, dass unter verwandten Elementen im Uralischen (und darüber hinaus im Indo-Uralischen) auch solche vorkommen, die sachlich kaum Wanderwörter sein können, jedoch auch in "alarodischen “ Gebieten auftauchen.

Die alarodischen Stämme sind gewiss in nahem Zusammenhang mit den Völkern, die nach den Ergebnissen der Bodenforschung an der grossen Völkerwanderung in der Zeit um $2500^{\mathrm{a}}$ teilnahmen, welche viele Völker in d. Richtung ost-west aus Zentralasien zum Mittelmeer und weiter nach Spanien (Iberer $=$ Baskisch) bis zum atlantischen Ozean brachte. Ein grosser Teil dieser Völkermassen wird später zu Indogermanen werden; ein anderer zu Uraliern usw. Schon mehr in Mitteleuropa (neben Raeti) die Etrusker von den Zentralen Alpen, weiter nach Zentralitalien (Etrüria); auf diese Weise wird allmählich nördliche Mitellmeerküste schon zu Beginn der Bronzezeit ca. $2000^{\mathrm{a}}$ ganz besiedelt, jedoch so, dass ein recht grosser Teil der neuen Ankömmlinge (zunächst Syrien, südliche Küste von Kleinasien, Griechenland mit Ägäis, Italien, Sizilien u.á.) indogermanisiert war; das bedeutet, dass die Indogermanen, die gewiss mit anderen Völkern verbunden nach Westen reisten, auch sprachlich ziemlich gut die Sprachen der Mitreisenden kannten. Unter diesen Umständen und vor allem wegen der Tatsache, dass sie sowie die Mitreisenden die- 
selbe Sprache benutzten, diejenige der Urheimat, dank den Ähnlichkeiten zwischen den Sprachmitteln der Indogermanen und ihren Nachbarn konnten sich gut verständigen, konnten auch von den nachbarlichen Völkern hie und da gewisse Elemente der Nachbarsprachen sich aneignen. Auch wenn die Sprachen der neuentstehenden Volkssplitter schon recht stark veränderte Mundarten sprachen, wären die entlehnten Wörter noch immer sich ähnlich, d. h. Entlehnungen und einheimische = ererbte Wörter waren zunächst noch in neuer Heimat gewissen Völkern sozusagen gemeinsam. Vgl. etr. laut-n- "Familie" zu idg. *leudh- "wachsen, Kind" POK. 684 f; etr. Tin-ia "Iuppiter" zu idg. *di-no- "Tag" s. POK. 186; etruskische Fälle derart sind noch mehrere vorhanden, darum ist Kretschmers "Protoindogermanen" ganz am Platze. In grosser Liebe zur Herkunft der Etrusker ist also OŠTIR mit seinem Gegner einig.

So war es in jener Epoche: ein Indogermane konnte ohne Dolmetscher einen späteren Uralier usw. gut verstehen; und in folgenden 1000 Jahren ist die geographische Differenzierung so stark geworden, dass wirkliche Sprachen enstanden. Die nicht immer folgerichtigen Entwicklungsprozesse liessen früher jedoch wahrscheinlich zahlreiche Restwörter in den Nachbarsprachen, die weiter nach Gesetzen neuer Umgebung Entwicklungsrichtung einahmen: so mit idg. - dh- etr. lautn- oben; Aetna, rutilus u.a. Ich bemerke hier, dass diese Lautwandel keineswegs auf "pelasgischen" Ideen von van WINDEKENS beruhen, da ich auch mit eigenem Gehirn neue Hypothesen und Theorien zu formen weiss; Obiges ist von v.W. nicht einmal flüchtig beeinflusst worden.

Unsere Pflicht ist dabei, das ganze von OŠTIR gesammelte Material neu zu prüfen, d.h. alle OSTTIRS Texte vollständig zu edieren; einen erschöpfenden Kommentar zu schreiben und dazu einen Index verborum hinzuzufügen.

OŠTIRS Gewohnheiten waren in gewissen Kleinigkeiten etwas bizarre. So war er im ganzen Leben nur bei zwei Doktordissertationen der Vorsitzende zu sein bereit. Im Falle B. C̆oP weigerte er sich das zu tun, trotzdem dass der Inhalt die Laryngaltheorie (dies betraf ja ihn persönlich) mit indouralischer Verwandtschaftstheorie (= idg. $V H V=$ ural. $V k V$ ) verband.

Es gibt mehrere Gründe dafür, dass die sprachwissenschaftliche Öffentlichkeit viele seiner Ideen mit Missgunst, Abneigung, ja sogar mit Spott zur Kenntnis nahm. Die Kritiker wendeten dabei manchmal Ausdrücke wie: unwahrscheinlich, abwegig, phantastisch, abzulehnen usw. an. Freilich gilt das nur für seine Alarodier. Die grosse Mehrheit hielt sich fern von diesem Streit; es gab ja andere ausgiebigere Gebiete zur sprachwissenschaftlichen Betätigung; dazu gesellten sich neue Fundorte, die neue Texte mit neuen oder besser belegten Sprachen und Sprachgruppen boten, zu. Neben das gerade phantastische Reichtum der archäologischen Funde traten philologische und sprachwissenschaftliche Aufgaben, neue Angaben, die uns besser zu belehren imstande waren. Die Alarodistik musste auch anders werden oder anderen Platz abtreten! 
Das musste für OŠTIR fast eine Katastrophe bedeuten! Als er noch ganz jung war, stellte er sich die Aufgabe, das sog. Alarodisch und seine Rekonstruktion zum Lebensziel. Diese Zielsetzung war bei ihm so stark, dass er ihr immer, auch in reifen Jahren, den Vorrang gewährte; um es sicherer zu machen, nahm er sich Zeit, um auch Archäologie und Ethnologie u.a. adäquate Fächer kennen zu lernen*. So wurde er zu einem Eruditus, den auch seine Gegner hochschätzten. Diese schwierige psychische Lage führte allmächlich einen starken Pessimismus und Zynismus herbei. Davon eine totale Abneigung gegen das Publizieren eigener Ideen. Die letzte seiner Schriften war "Drei vorslavisch-Etruskische Vogelnamen" (Ljubljana 1930, 112 Seiten). Inzwischen entwickelte sich bald nach 1930 eine neue sprachwissenschaftliche Richtung, die Indo-Uralistik, welcher u.a. B. Čop seine Kräfte in immer grösserem Masse widmete; vgl. seine Publikationen: Dekl., Laryng. 1970, Indouralica $I-X I X$ und viel anderes. OšTIR liebte diese Neuigkeit allerdings nicht, obwohl er auch Ideen dazu lieferte; vgl. mordw. o- $m b o$ "ander" = idg. *á(m)bhō(u) "beide" in Orbis $X X I I$ (1973), S. 22. Ein weiterer Ausdruck seiner Verzweiflung und Verzichtung war "Ach, Mist, noch schlechter als das von mir!", was einem Armenologen galt. Und doch: Als in letzten Jahren OŠTIR eine der neuesten alarodischen Abhandlungen vor Augen bekam, sagte er mit tiefem Seufzer: "Jetzt tut es mir wirklich leid, dass ich nicht mehr jung bin!“. Das Wirken von OŠTIR blieb durch seine Schriften und mündliche Mitteilungen bis auf heute beachtlich; doch gingen einige seiner Freunde in der Nachahmung der Alarodistik viel zu weit (so ŽUPANIČ in Etnolog). Hier ist am Platze zu sagen: Alles ist mit allem verbindbar (natürlich ironisch). OšTIR fühlte klar dieses Stilem und zog langsam mit seinem etwas wilden System zurück; vgl. Vogelnamen in Gegensatz zu den älteren Werken. Auch wurde das Sumerische immer vorsichtiger herangezogen, wahrscheinlich wegen der philologischen Schwierigkeiten dieser Sprache, die der junge OŠTIR in jugendlichem Schwung niederschluckte, während der alte bei aller Vorsicht es lieber beiseite schob. Wir, seine Nachfolger, rari nantes in gurgite vasto, schätzten unseren wohlwollenden Lehrer und Ermahner ebenfalls hoch. Ihm gebührt unsere tiefste Dankbarkeit für alles, was er für uns und für unsere spracwissenschaftliche Zukunft getan hat. Er lebt in unserem Geist, ein kleiner Mann, mit Pfeife in der Hand, langsam, vorsichtig schreitend, in tiefe Gekanden versunken, sicher in der Problematik der alarodischen Sprachen grübelnd.

Mit diesem Bilde dürfen wir unserer Rede ein Ende machen.

Nur soll es nicht vergessen werden, dass in unseren Ausführungen die ältest belegte nostratische Sprache mehrmals erwähnt wurde: das Sumerische. Ich stelle diese Sprache zu den nostratischen, schon gut bekannten sieben Sprachstämmen: idg., ural., alt., ham.-sem., süd-kauk. und drav.; vgl. ILlıČ-SvITÝč, Opyt I-II (1971-1976); er bringt etwas mehr als 350 Etymologien, wohlgemerkt nicht immer aus allen sieben bei jeder Gleichung, wie auch zu erwarten war! Ich stelle meinerseits noch die achte diesmal isolierte Sprache hierher, nämlich das Sumerische; die fol-

* (Meringers Schule). 
gende Liste (16 Stück) bietet allergewöhnlichste Methode, und doch könnte sie vielfach vermehrt werden. Abkürzungen: wie in der indogermanistischen Literatur. $\mathrm{Na}$ tionalität der Wörter: SU, IG, UR. Ich folgte nur meinen Ideen; die Ph in SU recht unsicher, ich folge der Transkr., die meistens in Gebrauch ist.

1. SU P/BÀD "Wall, Befestigung"; lat. periës "Wand, Zwischenwand"; UR finn. pato "Damm, Wehr, Wall", lapp. buo $\delta \delta o$ usw. nach B. COP, $K Z$. 88, 1974, S.

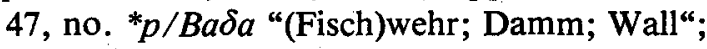

2. SU BURU "Loch; brechen, stechen" zu IG lat. forāre, ahd. borōn; UR finn. + pura "Bohrer"; die Sippe bei POK. 133-135 hat natürlich unsere Sippe historiographisch aufgesogen; man muss die beiden voneinander trennen, wie der Vokalismus zeigt ( $-u-$ in UR und SU).

3. SU DÜ "machen, tun" zu IG *dhe- "setzen, legen, stellen; machen, tun", aind. $d h \bar{a}$ - usw. POK. s.v.; UR finn. teke- "machen, tun";NO *Deke-, im UR die Urbedeutung zum Teil verloren.

4. SU KAL, KALAG, KALAGGA "stark, mannhaft" in zwei idg. Sippen: a) "hart" z.B. sl. kaliti (Eisen); b) "stark, gesund" z.B. ahd. helid, aind. kalyá-, gr. kalós (a +b Pok. 523-524). NO *kala "hart; stark, gesund“.

5. SU KƯ(G) "hell, glänzend, rein" erinnert an eine starke Gruppe von Farbwörtern bei POK. 564f.: a) idg. *kenakó- "gelblich, saflorfarben", u.a. d. Honig samt Sippe; auch aind., apreuss.; gr. knākos, knēkos "Art Saflor"; b) Urwort NO *kuךe "Mond" in UR finn. kuu id. (sek. "Monat" usw.), alt. + $r$-Sx. Die ganze Sippe (auch SU) bei ČP, UAJb. 44 (1972), S. 289. Zur $P h$ an e. anderen Stelle.

6. SU LUH "waschen; spülen": dazu IG $* l o H-$, erweitert $* l o H-u$ - ds. in heth. laH-, lahva-, la(h)hu- “ergiessen“ nur mit $-u / w-:$ lat. lavō, gr. lówō usw. (s.B. C̆OP, Laryng. 1970, S. 193/11).

7. SU NU:"nicht", zu IG *ne ds. (aind. ná, sl. ne + bei POK. 755-578), zum Vokal im SU vgl. Nr. 2 BURU, Nr. 3 DÙ, Nr. 14 TÚG; das UR und Alt. haben gewöhnlich ein Verbum negativum. Spuren desselben auch im Idg., s. IU. IX (im Druck).

8. SU MÁŠ "Familie, Sippe; Nachkommenschaft", auch in Verbindungen wie: máš gal "königliche Familie", máś lú "männliche Verwandtschaft", máš sal "weibliche Verwandtschaft", ša máš "(der) der Verwandtschaft, Verwandter", nach B. ČOP zu der Sippe finn. mies, miehe- "Mann", vog. mo(ó)s'- "der Name éines von den zwei exogamischen Klans", dasselbe in Ostj. (FUV. ${ }^{2}$ S. 114). Ural. + SU noch + lat. mās, Gen. măr-is in Die Sprache 22 (1976), SS. 25-28; im IG noch ai. pú-māñs-.

9. SU ŠE “Korn, Getreide“ (als Lehnwort akkad. še'u); hiedurch kommt man zu einem ureurasischen Getreide-Wort *säntä (Wichmann, FUF. XIV (1914), SS. 18-19; später + ung. ed "Getreide", ostj. DN tänt "Gerste; Getreide"; + ostj.; čer. š- usw.; zur ganzen Gruppe von *šäntä vgl. noch B. C̆OP, Lingu. XVI (1976), SS. 18-19. Details anderswo. 
10. SU SAH "Schwein", geht wahrscheinlich zu IG *sū: $-s=$ gr. $h \tilde{y}-s$, germ. ${ }^{*} s \bar{u}-z$. $=\mathrm{d}$. Sau, evident alle aus laryngalhaltigem ${ }^{e}{ }^{e} \mathrm{Hu}-\mathrm{s}$ usw., der theoretische Laryngal ist nun selbst ans Tageslicht getreten. IG POK. 1038-1039. - In irgendwelchem ähnlichem historischem Verhältnis dazu steht UR finn. sika "Schwein", mdw. tuva usw. ("tika $+S x-u$ - wäre genau das Obige IG).

11. SU TÚG "Kleid" nach B. C̆OP, KZ. 84 (1970), S. 163 (lapp., sam.; mordw.

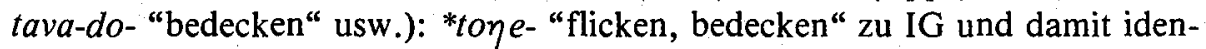
tisch = lat. tegō usw. NO *têך $\hat{e}$ - "flicken, bedecken".

12. SU TÙR (tarbasu) "Viehhof"geht wohl zu idg. *twer- "fassen, einfassen, einzäunen“, z.B. bei POK. 1001 vgl. lit. tvorà "Zaun“.

13. SU UDU "Schaf" gehört m.E. zu gleichbedeutendem finn. uuhi, Var. uute usw. "Schaf" (in mehreren Varianten belegt, deren Ausgangsbildung scheint mdw. $u \check{c} a$ id. darzulegen, aus urmdw. *ütj^ -: finn. *üte- usw., im SU ein $u$-Stamm(?); ein Typus mit kurzem *ŏtj- kommt später zur Sprache. Vgl. Toivonen, FUF. $X I X(1928)$, Nr. 7.

14. SU UR "männlich (Mensch)“ Männchen (grosse Raubtiere)", auch in Verbindungen, wie: ur.zir "Hund" ur.mah "Loewe" ur.barra "Wolf", ur.sag "Held": Völlige Deckung mit finn. uros bei FUV. ${ }^{2}$ S. 134 "male“, ung. úr "Herr, Herrscher", finn. noch "erwachsener Mann, Held".

Ich könnte hunderte solcher Gleichungen vorführen; ich erwähne nur noch, dass in Obigem (und sonst) ungewöhnlich oft auf beiden Seiten eine oder zwei Silben mit Vokal - $u$ - (wahre Aussprache?) stehen. Ein solches UR-Wort soll auch in SU GU(d) "Rind", idg. ${ }^{*} g$ " $\bar{o} u$ - stecken. Jedoch macht das $-d$ bedenklich, weil die Urform auf $-d$ auslautete. Phonetisches oben unter š Nr. 9. Eine Parallele aus der Grammatik in folgenden Nummern $(15+16)$ :

15. SU EN "Herr", m.E. aus dem Wort für "Haus", SU $e$, und einem Suffix $-n$ "Herrscher über.."; vgl. u.a. lat. domi-nu-s zu domu-.

16. Wohl mit obigem ist das Gen. $-S x *-n$ des UR und IG, identisch, vgl. meine Deklination (1975), SS. 53-55; vgl. gr. $-n$ in /argyro- $n$ /, heth. - $a-n$ in Labarna- $n$ u. ähnliches / UR in čer. $\beta \ddot{u} \delta \gamma-n$ "des Wassers", finn. koda-n "des Hauses" SU, erhalten in romantisch anmutendem Juxtapositum geš- $t i-n$ "Wein" $=$ wörtlich "der Baum" (= geš-, sonst giš) *ti-n "des Lebens" ( $t i$ SU "das Leben") $+-n$, einstige Endung der nominalen Genitivform; begrifflich vgl. frz. eau-de-vie.

Auch durch diese 2 Restelemente erweist sich das SU als eine nostratische Sprache. - Dieser kleine Beitrag aus der Sumerologie würde OŠTIR Gefallen tun und Ansehen schaffen, da ja der Autor sein Lehrling war. OŠTIR wollte in seinem Spezialfach frei von aller fremden Suggestionen bleien.

Mit allem Obigen beladen konnte nun unser Professor nicht verhindern, dass ein Sprachwissenschaftler, der ihn gut kannte, ihn an einem Kongress das grösste Original aller Zeiten nannte. Nun, da die Rede zu Ende ist, können wir sagen: 


\begin{abstract}
Alle, die OŠTIR kannten, sahen in ihm eine Persönlichkeit, die lieblich war jedoch weder spöttisch noch heuchlerisch, Wahrheit und Freiheit über alles andere schätzend, korrekt gegenüber den Kollegen, rechtschaffen und redlich in ganzem Leben, als Wissenschaftler im Besitz enormes Wissens sowohl in sprachlichen Dingen als auch in anderen anthropologischen Fächern, die alle um ein Zentrum, den Mensch, sich tummeln. Aber als Opfer eigener Hartnäckigkeit auch eine tragische Person, die ihre Lebensaufgabe erfolgreich anfing, aber mit einer Absagung endete. Seine positiven Taten - viel zahlreicher als negative - bleiben für ewig in Erinnerung und schriftslichen Traditionen.
\end{abstract}

"NON OMNIS MORIAR", sagte von sich einer der grössten Poeten. Nun, ist die Sprachwissenschaft nicht auch höchste Poesie?

Povzetek

OB STOLETNICI ROJSTVA PROF. DR. KARLA OSTTIRJA

Oddelek za primerjalno jezikoslovje in Lingvistični krožek Filozofske fakultete v Ljubljani sta proslavila 100 letnico rojstva prof. dr. Karla Oštirja; sicer so slovenski jezikoslovci imeli do zdaj dovolj priložnosti, da se spoznajo tudi po Oštirjevi smrti z marsikatero čudaško potezo tega moža (n.pr. v Linguistici XIII). Za to obletnico smo pripravili rajši nekakšen pregled čez Oštirjevo duhovno rast in njegove odnošaje do drugih učenjakov, ki so mu nasprotovali, ali pa takih, ki so mu slepo sledili. Tu smo opozorili na vlogo sumerščine, predvsem seveda na ves Vzhodni Mediteran. Marsikatera stvar je bila izrečena ironično, tako da je Oštir brez svoje krivde zaslovel kot največji original na vsem jezikovnem področju. 